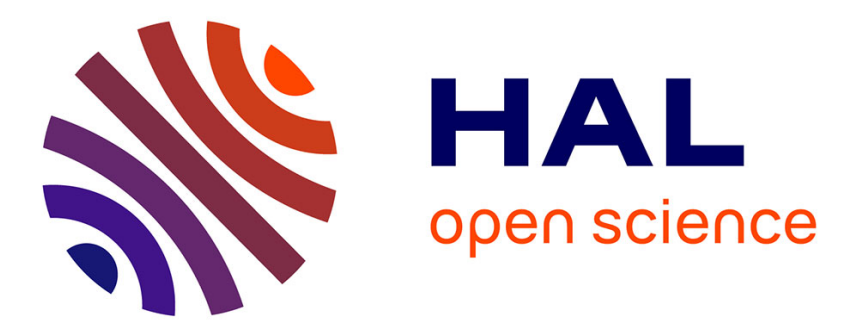

\title{
Sensitivity approach for modelling the environmental loading of marine structures through a matrix response surface
}

Franck Schoefs

\section{- To cite this version:}

Franck Schoefs. Sensitivity approach for modelling the environmental loading of marine structures through a matrix response surface. Reliability Engineering and System Safety, 2007, 93 (7), pp.10041017. 10.1016/j.ress.2007.05.006 . hal-01007280

\author{
HAL Id: hal-01007280 \\ https://hal.science/hal-01007280
}

Submitted on 16 Jun 2014

HAL is a multi-disciplinary open access archive for the deposit and dissemination of scientific research documents, whether they are published or not. The documents may come from teaching and research institutions in France or abroad, or from public or private research centers.
L'archive ouverte pluridisciplinaire HAL, est destinée au dépôt et à la diffusion de documents scientifiques de niveau recherche, publiés ou non, émanant des établissements d'enseignement et de recherche français ou étrangers, des laboratoires publics ou privés.

\section{(c)(1)}

Distributed under a Creative Commons Attribution| 4.0 International License 


\title{
Sensitivity approach for modelling the environmental loading of marine structures through a matrix response surface
}

\author{
Franck Schoefs \\ Nantes Atlantique University, CNRS, Institute in Civil and Mechanical Engineering (GeM), UMR 6183, Faculté des Sciences et des Techniques, \\ 2 rue de la Houssinière, Nantes F-44000, France
}

\begin{abstract}
The actual challenge for the reassessment of existing offshore structures through a rational process of reassessment leads one to state the importance of a response surface methodology. At different steps along the quantitative analysis, quite a number of approximations are developed as surrogates for the original model. They require subsequent uncertainty and sensitivity studies. This paper suggests a geometrical description of the energy of the wave kinematics fields for the building of a wave loading response surface. The matrix response surface of the water particle kinematics field is shown to be suitable for further transfers and for uncertainty and sensitivity studies. This allows discussion of the complexity level to be introduced in stochastic modelling of the wave actions within its relevance for the probabilistic mechanics analysis of offshore structures. For illustration, in situ measurements are used to provide ranges and distributions of basic variables with a view to performing uncertainty and sensitivity studies.
\end{abstract}

\section{Introduction}

Nowadays, one third of the existing offshore platforms require life extension. It is well recognized that the probabilistic reliability approach gives an efficient quantitative means for updating additional information and for measuring the relative change in safety level compared with a predefined requirement. During this last decade, efforts were concentrated on developing methods and their corresponding software for structural reliability analyses. More and more in recommended practice, resulting failure probabilities and/or reliability indices are introduced for elaboration of LRFD rules and comparison of design proposals. The actual challenge lies in providing rational procedures and decision aid tools related to the reassessment of offshore structures where the structural and mechanical integrities are important criteria.

The required structural behaviour can be considered as the output of a system, which varies in response to the changing levels of several input variables. The response

\footnotetext{
E-mail address: franck.schoefs@univ-nantes.fr
}

surface methodology (RSM) comes, of course, as a basic formal aid tool. The challenge is that, starting from environmental loadings and resistance factors in order to obtain reliability indices, the models are to be provided meaningfully. Therefore, the response curves must be based on some prescribed understanding of the underlying mechanism [1]. Key factors and underlying concepts to control RSM are introduced in Section 2. The primary concern is about how to provide criteria for rational guidance when choosing a response function format among potential candidates.

Section 3 presents the step-by-step building of a matrix response surface of wave loading on framed offshore structures that is based on the energy of the wave kinematics fields. The main transfer concerns the kinematics field on which all the response surfaces are based. The time evolution of the wave propagation is usually described by second-order partial differential equations within more or less simplified boundary and initial conditions, known as Navier-Stokes equations. Useful reviews of wave loads acting on offshore structures are available in a series of articles [2-22] including the effect of 
currents or marine growth. The main focus is on deterministic aspects even if effect of uncertainties in marine growth modelling for example is available [22]. In terms of structural reliability analysis, probabilistic modelling is mainly based on polynomial response surfaces. This paper presents a general framework and methodology to deal with uncertainties in a complete chain for computing nodal forces. It starts from a formal representation of the wave kinematics field, following a geometrical viewpoint. The $n$ th-order Stokes model has the form of a sum of $n$ vectors, each of them being obtained through suitable similarity and rotation transformations of the wave direction vector. The similarity ratios and the rotation angles are random functions. Their stochastic fluctuations depend on wave height and wave number. This leads to a specification of the random nature of the wave kinematics intensity process. Subjects such as physical meanings, complexity level in modelling, distributions effects and computational tractability are carefully addressed in Section 3.

The response surface based on geometrical description of wave kinematics is shown to be very tractable for sensitivity and uncertainty studies. It is a good basis in order to build the complete response surface of energetic equivalent nodal forces. Successive mathematical developments in Section 4 are devoted to this aim.

\section{Probabilistic analysis for marine structures design and reassessment}

\subsection{Identification of major structural behaviours}

Consider an offshore structure buffeted by waves and also submitted to wind and current loads. Due to the unsteady and random nature of environmental forces and the imperfections of structure and materials, the technological risk due to a collapse cannot be absolutely eliminated. This leads to the conclusion that safety must be quantified, and risk must be evaluated through measures and finally compared with an advocated risk level. Under these considerations it is now widely accepted that the probabilistic mechanics approach gives an efficient means for conducting a reliability analysis. Concepts, tools and software [23] have been developed during the last 20 years for the evaluation of strength, load variables and reliability measures.

The mechanical behaviour of structures embraces usually three major loading types, which are extreme events, fatigue and dynamic loading. Practically an analysis of the structural shape and the platform location becomes a rational guidance to select the dominant loads, which defined the first design of the structure. In deep water for example, a tower is suggested to dynamic excitation. With regard to a process of reassessment for tiny structures called jacket platforms, which are in fact memory-less and non-resonant structures, structural response to extreme events and fatigue loading has to be quantified. This paper focuses on the first case: quasi-static behaviour is then considered.

\subsection{Specific needs for structural reassessment}

The safety margins of offshore structures vary from the design stage and during the service life due to several parameters evolving during the life of the structure (marine growth, technical change, etc). The reassessment of existing platforms is a particular stage where these factors are to be updated from survey programmes and measurements of environmental conditions. It allows measuring the relative change in safety level compared with a predefined requirement. The collected data contribute to a better knowledge of structural and environmental properties. One must keep in mind that the uncertainty is not automatically growing with time. For example, the marine growth effects can be affected by the transport and the period of implantation of the structure, and the corresponding uncertainties are larger at the design stage than after several years of structural survey.

\subsection{Motivation for the building of a physical response surfaces}

As a consequence, analytical expressions of strength, load variables and limit state function are to be proposed. Classically, these surrogates to original models are derived from a regression analysis. There is no hope to define a general procedure for the selection of an approximation format among all the possible candidates. It leads one to state the importance of the RSM [1] as a basic formal aid tool. It is important to notice that these derived functions (mainly polynomial expressions) are generally used as the simplest readily available smoothing curves, without any appeal to their theoretical properties as asymptotic approximations to the true response function. Leira et al. [24] show that even the expectation of the response can be erroneous using polynomial expansions with a low degree. Such a "blind approach" is very questionable [1]. Here an alternative procedure for constructing response surfaces is suggested, which combines two guidelines, a deterministic approach in which the successive transfer functions are derived from physical considerations with the stochastic modelling of the basic variables. The first deals with the efficiency of the deterministic models themselves. The second, on the basis of uncertainty and sensitivity studies, needs to identify the basic random variables, e.g. those that govern the variations of physical quantities.

Clearly, the objective is not to improve new deterministic models but to suggest a rational process of response surface modelling based on uncertainty and sensitivity studies (see Section 4). 


\section{Building a response surface for describing wave loading}

\subsection{Guidelines and key points of the response surface methodology}

RSM has been largely developed during the last decade, and response function models, including non-linear ones, are now available, especially for the specific needs of structural reliability analysis [25-28] and stochastic problems such as crack propagation [29]. This methodology is basically a formal representation based on geometrical ideas: surface building in the probabilistic space. It leads to investigation of the properties of a physical process versus a system approach. This means that the required response (e.g. mechanical behaviour) is considered as the output of a system, which varies in response to the changing levels of several input variables (stimuli) called basic variables (see Fig. 1). The system must be modelled by some mathematical function of the random variables $X_{i}$ involved in the system and characterized by statistical information $\theta_{i}$ (moments, free or parametric distribution functions). For reliability analyses, the investigated response is either strength, load variables or limit state function. The surrogates to original models are classically analytical functions (mainly polynomial expression) whose parameters are estimated from experimental results $[26,27,30]$. The building criteria must be developed according to the specific reliability analysis. Three major criteria, which may conflict, have been proved to be relevant [31]:

- Physical meanings: The selection of the set of stimuli and of the approximation function is expected to be meaningful in the sense that it is based as far as possible on some understanding of the underlying mechanism of the physical process.

- Distribution effects: The output distributions change according to the various assumptions selected to describe the statistical properties of the stimuli variables $X_{i}$ and to fit the function $f$. The selection should reflect how the whole distribution is well transferred through the response surface (the so-called distribution effects) especially for non-linear transfer such as the transfer of the energy of the wave kinematics field. It is proposed here to ensure the main distribution effects by the additional fitting of the partial derivatives of $f$ relatively to $X$, i.e. the Jacobean matrix.

- Goodness-of-fit measure: Let us consider a response function and its robustness in an attempt to perform inference analysis. The first concern is the selection of

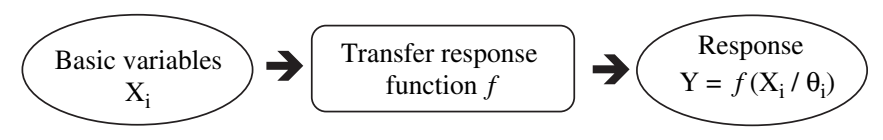

Fig. 1. System modelling using response surfaces. the basic variables $X_{i}$ and specially the ranking of them. The usual metrics termed sensitivity and uncertainty importance measures are less efficient. According to the fitting demonstrated in the previous subsection, the metrics of Sobolev space [32] can be suggested to introduce some mean least-squares error on the partial derivatives as a measure of the goodness of fit. That largely conditions the safety domain topology and its probability measure. This approach questions the choice of a polynomial response surface of low order, so suitable for the computational implementation [1].

- Computational tractability: Clearly the complexity reduction and computational tractability are incentive requirements for engineering purposes. It is observed that increasing use and development of electronic computers and algorithms offer important potentials in this area. However, an increase of the complexity level in stochastic modelling does not necessarily ensure more realistic results and is always a time-consuming option when computing.

Alternatively to the regression techniques and according to these different criteria, this paper suggests a procedure for building response surfaces, which combine the stochastic modelling of the basic variables with a deterministic approach in which the successive transfer functions are derived from physical considerations. This self-consistent approach based on sensitivity and uncertainty studies enables ranking of the input variables and to well disseminate the associated statistical assumptions. It gives arguments for the ability of the selected models to be used for inference purposes. The illustration presented below concerns the transfer of the energy of the wave kinematics fields for the calculation of wave loading on a steel framed offshore structure called a jacket structure.

\subsection{Building the response surface of energetic equivalent nodal loads}

\subsubsection{Kinematics of wave loading}

Considering a steel framed structure submitted to wave and current, the source of the resulting environmental loading resides in several factors such as the group effect of piles, welding and building conditions, and soil-structure interaction. The focus here is on the kinematics of water particles near the wet structural component. For the reassessment process of non-resonant structures, it is noticed that fatigue and quasi-static structural behaviour are of primary concern. For structures showing this behaviour, the environmental loading is considered through its static effects and based on the wave kinematics. There is no hope to define a general procedure to select the corresponding model and the associated response function among all the candidates available. The choice of format and complexity level must be well argued in the sense of its consistence versus the global approach of the problem (probabilistic and mechanical modelling for 
reliability analysis). For the presented study, according to the specific response surface criteria presented above, several targets that may be in conflict are taken into consideration:

- The model is required to be meaningful in the sense that it is based as much as possible on some understanding of the underlying mechanism (physical major considerations of the wave-structure interaction): wave-current interaction around the beam (vortex shedding) especially in the presence of mixed marine growth (soft and hard).

- One must justify its consistence as a link into the complete transfer between the previous one (wave kinematics) and the next one (computing of energetic equivalent nodal forces). To this issue a model based on energetic consideration is well adapted.

- Its complexity level is wished to describe the phenomena without a reasonable number of numerical computations (computational tractability).

- It should be used for inference purposes and suitable for the environmental conditions updating from inspections reports (marine growth evolution).

It must be added that the validation of the final format of the response function to the issue of its practice in reliability analysis must be argued on the basis of uncertainty and sensitivity studies.

To set up a computational model, equivalent concentrated forces applied at the nodes of the structure must replace the loads exerted by the waves. Schematically, successive transfers are described in Fig. 2. From the criteria exposed above, it is necessary to select a physical model of the force per unit span being based on energetic consideration. The so-called Morison equations are well adapted to this issue [33]. Their expression, translated in local coordinates, indicates that the force per unit span can be separated into drag (Eq. (1)) and inertial (Eq. (2)) components (Fig. 3):

$f_{\mathrm{D}}(M)=\frac{1}{2} \rho \theta_{\mathrm{mg}} D\left[\begin{array}{ccc}\pi C_{\mathrm{X}} & 0 & 0 \\ 0 & C_{\mathrm{D}} & 0 \\ 0 & 0 & C_{\mathrm{D}}\end{array}\right] \vec{V}(M)\|\vec{V}(M)\|$,

$f_{\mathrm{I}}(M)=\frac{1}{4} \rho \theta_{\mathrm{mg}}^{2} D^{2}\left[\begin{array}{ccc}C_{\mathrm{X}}^{\prime} & 0 & 0 \\ 0 & C_{\mathrm{M}} & 0 \\ 0 & 0 & C_{\mathrm{M}}\end{array}\right] \vec{\Gamma}(M)$,

where $\rho$ is the water density, $D$ is the cylinder diameter, $\vec{V}(M)$ and $\vec{\Gamma}(M)$ are, respectively, the instantaneous flow velocity and the acceleration at point $M$, and $C_{\mathrm{X}}, C_{\mathrm{D}}, C^{\prime}{ }_{\mathrm{X}}$, $C_{\mathrm{M}}$ are hydrodynamic coefficients (respectively two drag and two inertia coefficients) for modelling the fluid-structure interaction. They are introduced as model uncertainty variables; $\theta_{\mathrm{mg}}$ acts as a multiplier for taking into account the uncertainties of "marine growth" effects through a random increase of the beam element diameter (screen effect).

These equations generally predict the main trends in measured data quite well, once appropriate joint distribution for hydrodynamic coefficients and for $\theta_{\mathrm{mg}}$ is provided. The first one depends on sea-state parameters and the second one varies with marine growth nature [22]. Nevertheless, they are not able to translate all the complexity of

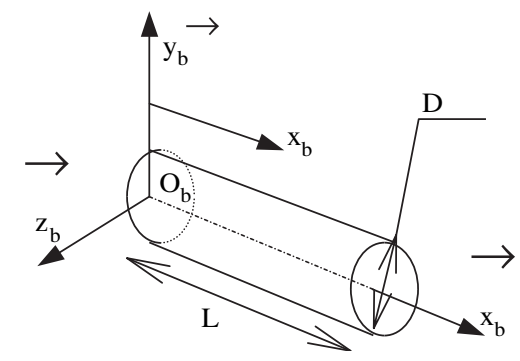

Fig. 3. Local coordinates $\left(O, \vec{x}_{\mathrm{b}}, \vec{y}_{\mathrm{b}}, \vec{z}_{\mathrm{b}}\right)$.

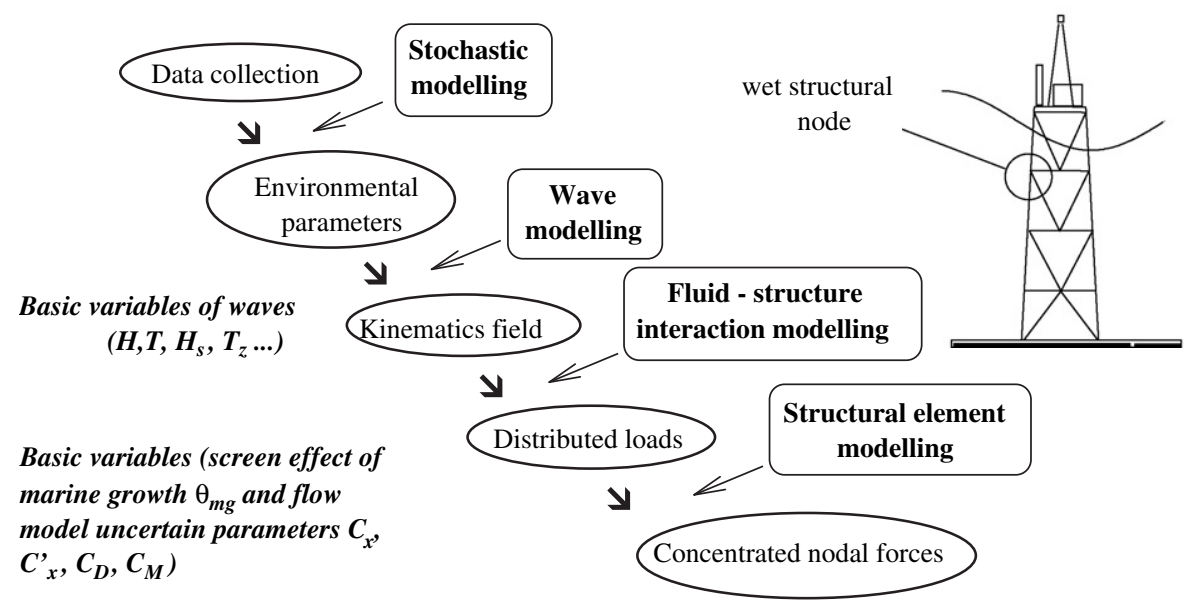

Fig. 2. Successive response surface modelling for concentrated nodal forces assessment. 
the fluid-structure interaction with enough accuracy (i.e. high frequency content of the flow, gross vortex shedding effects, etc.).

Keeping in mind these shortcomings as a source of response problems, they are relevant in introducing nonlinear effects and their complexity is consistent with respect to the large uncertainty in marine growth or fluid interaction modelling caused by the lack of investigations. This matrix expression leads to the proposal of a specific geometrical formalism for kinematics field modelling.

\subsubsection{Stochastic modelling and kinematics field formalism}

To compute the hydrodynamic forces on the structural components, there is a need to provide the kinematics field of fluid particles based on sea conditions. The specific interest of Eqs. (1) and (2) resides in the separation of two terms, linear and non-linear, and of the matrix format. This geometrical format can be very useful to perform physical analysis on transfer quality. The work on the kinematics field format has been based on this consideration. Moreover, its stochastic formalism should make the study of its trajectories easy to ensure the physical understanding of the transfer of its energy.

Physical time scales of wave actions are taken into account with practical efficiency, by identifying sea states as stationary components of a piecewise second-order stationary ergodic and differentiable in mean square random process [34]. As an extension of generalized harmonic analysis, the stochastic process theory is considering functions indexed by space/time parameters and with values in a so-called complete probability space [34]. One way of describing a stochastic process is to specify the $n$-dimensional joint probability law whatever the value of $n$. Another means is to give an explicit formula for the value of the process at each index point in terms of a family of random variables whose probability law is known (e.g. trajectories). The last option is chosen to describe wave-bywave actions on jacket platforms which are basically memory-less and non-resonant structures, especially because of their tractability for use in a probabilistic mechanics push-over analysis with computer programs based on finite elements.

To this extent a formal geometrical representation of the wave kinematics is developed with special emphasis on Stokes waves. This model describes waves with a rigid progressive wave profile without the small-amplitude assumption, and the fluid motion is irrotational with open orbits. It depends, as for all usual wave velocity models, on the random variables $H$ and $T$. This couple of basic variables is sufficient in the sense of the physical meanings criteria: it can be considered as summarized information on wave energy, which is consistent with the physical understanding of the Morison equations. For the study of the quasi-static structural behaviour, only extreme sea states (storms) are considered here and, in this case, these variables represent the extreme wave height and the conditioned period.
Let $(O, \overrightarrow{O U}, \overrightarrow{O Z}, \overrightarrow{O V})$ be a canonical repair in the Euclidean space $R^{3}$. The origin $O$ is taken at the mean sea level, the axis $\overrightarrow{O Z}$ is vertical and upward, and $\overrightarrow{O U}$ is the wave directional axis. The following set of time and dimensionless in space indices is considered:

$t, x=U / l, \quad z=Z / d+l$,

where $l$ is the width of the structure (diameter of a cylinder containing the platform) and $d$ is water depth.

The small-amplitude plane harmonic progressive waves known as Airy waves are the simplest of all solutions to the wave problem. They are derived from a velocity potential, also called orthogonal stream function. The associated velocity vector $\vec{V}_{\text {airy }}$ takes the classical form (see Eq. (4)) in the wave plane $(\overrightarrow{O U}, \overrightarrow{O Z})$ :

$$
\begin{aligned}
& \vec{V}_{(1)}=\vec{V}_{\text {airy }}=\frac{H}{2} \sqrt{\frac{g}{d}} \frac{\cosh (k d z)}{\cosh (k d)} \\
& \times \sqrt{\frac{k d}{\tanh (k d)}}\left[\begin{array}{l}
\cos \left(k l x-\psi_{t}\right) \\
\tanh (k d z) \sin \left(k l x-\psi_{t}\right)
\end{array}\right],
\end{aligned}
$$

where $H$ is the wave height, $k$ is the wave number deduced from the random period $T$ by the dispersion equation, $\omega$ is the pulsation and $\psi_{t}=\omega_{t}-\psi$ ( $\psi$ : phase angle). The variables $k$ and $\omega$ are linked together by the one-to-one dispersion equation relation $\left(\omega^{2}=g k \tanh (k d)\right)$.

The velocity vector being in the wave plane, it can be written in the geometrical form,

$$
\begin{aligned}
& \vec{V}_{\text {airy }}=\mathscr{H}\left(O, \lambda_{(1)}\right) o \Re\left(\overrightarrow{O V}, \alpha_{(1)}\right) \overrightarrow{O U}, \\
& \tan \left(\alpha_{(1)}\right)=\frac{\left\langle\vec{V}_{\text {airy }}, \overrightarrow{O Z}\right\rangle}{\left\langle\vec{V}_{\text {airy }}, \overrightarrow{O U}\right\rangle}=\tanh (k d z) \tan \left(k l x-\psi_{t}\right), \\
& \lambda_{(1)}=\left\|\vec{V}_{\text {airy }}\right\|=\frac{H}{2} \sqrt{\frac{g}{d} \frac{\cosh (k d z)}{\cosh (k d)}} \\
& \times \sqrt{\frac{k d}{\tanh (k d)}} \sqrt{1-\frac{\sin ^{2}\left(k l x-\psi_{t}\right)}{\cosh ^{2}(k d z)},}
\end{aligned}
$$

where $\mathscr{H}\left(O, \lambda_{(1)}\right)$ denotes the similarity with centre $O$ and ratio $\lambda_{(1)}$ and $\Re\left(\overrightarrow{O V}, \alpha_{(1)}\right)$ denotes the rotation of $\overrightarrow{O V}$-axis and with angle $\alpha_{(1)}$.

The similarity ratio $\lambda_{(1)}$ and the rotation angle $\alpha_{(1)}$ are random functions indexed by $(x, z, t)$. The fluctuations of their trajectories depend on the couple of random variables $(H, k)$. Angle $\alpha_{(1)}$ is analytically independent of variable $H$. The range and statistics of $k$ are well adapted to approximate the vector $\vec{W}=\Re\left(\overrightarrow{O V}, \alpha_{(1)}\right) \overrightarrow{O U}$ by the normalized first-order Taylor expansion $\vec{W}^{(1)}$ around the mean wave number $\bar{k}$. This point will be discussed in more detail in a following paper. Introducing inside Eq. (5) the approximation $\vec{W}^{(1)}$ instead of $\vec{W}$, the velocity field corresponding to the Airy model has the following 
response curve,

$\vec{V}_{\text {airy }} \approx \vec{V}_{\text {airy }}^{(1)}=a_{(1)} \vec{A}+b_{(1)} \vec{B}$

where $\vec{A}$ and $\vec{B}$ are deterministic orthonormal vectors defined by

$\vec{A}=\Re\left(\overrightarrow{O V}, \alpha_{(1)}\right) \overrightarrow{O U} \quad$ and $\quad \vec{B}=\Re(\overrightarrow{O V}, \pi / 2) \vec{A}$

and the coefficients $a_{(1)}$ and $b_{(1)}$ are random functions,

$a_{(1)}=\frac{\lambda_{(1)}}{\sqrt{1+\mu_{(1)}^{2}}}, b_{(1)}=\mu_{(1)} a_{(1)}, \mu_{(1)}=(k-\bar{k}) \underline{\alpha_{(1)}^{\prime}}$,

where $\alpha_{1}^{\prime}$ denotes the partial derivative of $\alpha_{(1)}$ versus $k$, at $k=\bar{k}$.

The random variable $\mu_{(1)}$ is zero mean and with narrow support. It follows that $b_{(1)}$ is small compared with $a_{(1)}$. Consequently, $\vec{A}$ represents the main axis for the velocity vector. The component following $\vec{B}$ leads one to consider the fluctuations of the velocity vector to be inside a narrow sector around $\vec{A}$. The $n$ th-order potential being the sum of a set of $n$ potentials, the response surface of each term $i$ is obtained by a similar transformation.

$\vec{V}_{i \text { th }}=a_{(i)} \vec{A}_{i}+b_{(i)} \vec{B}_{i}$,

where $\vec{A}_{i}$ and $\vec{B}_{i}$ are two deterministic orthogonal vectors in the wave plane. After projection of these vectors on $\vec{A}$ and $\vec{B}$, the response surface of the $n$ th-order wave velocity is deduced (Eq. (12)):

$\vec{V}_{n \mathrm{th}}=a_{(n)} \vec{A}+b_{(n)} \vec{B}$

The multipliers $a_{(n)}, b_{(n)}$ are analytical random functions. They are expressed as functions of the wave height and of the wave period. Thus their stochastic fluctuations are governed by the randomness of these basic variables. The expression of the acceleration has a similar formal representation. It is deduced from the differentiation in time of (12). Consequently, it is a linear combination of the same vectors within introduction of two coefficients $c_{(n)}$ and $d_{(n)}$ (see Eq. (14)). This geometrical format enables the introduction of (see Section 4) a stochastic structure following space/time indices. As an example, wave kinematics amplitudes are profile functions (stochastic variations with depth) mainly.

The complexity level is discussed when order $n$ is selected in the random functions $a_{(n)}$ and $b_{(n)}$. Eq. (12) makes this analysis easier since each term of order $n$ can be expressed as an additional perturbation of the previous order $(n-1)$. This item is illustrated in Section 4.3.

\subsubsection{Distributed loads and energetic equivalent nodal loads}

The geometrical format of the kinematics field response surface is well adapted to the matrix expression of the Morison equations. The response surface of the distributed loads on a wet beam is then calculated with an increase of the number and nature of basic variables only, from 2 ( $H$ and $T$ ) to 7 ( $H, T$, four hydrodynamic coefficients and the marine growth multiplier). The expression of response surface of drag (1) and inertial (2) components in local coordinates is deduced in Eqs. (13) and (14),

$$
\begin{aligned}
f_{\mathrm{D}}(M)= & \frac{1}{2} \rho D \theta_{\mathrm{mg}} \sqrt{a_{(n)}^{2}+b_{(n)}^{2}} \\
& \times\left[\pi C_{\mathrm{X}} \prod_{\mathrm{b}}+C_{\mathrm{D}} \prod_{\mathrm{b}} \perp\right]\left(a_{(n)} \vec{A}+b_{(n)} \vec{B}\right), \\
f_{\mathrm{I}}(M)= & \frac{1}{2} \pi \rho D^{2} \theta_{\mathrm{mg}}^{2} \\
& \times\left[C_{\mathrm{X}}^{\prime} \prod_{\mathrm{b}}+C_{\mathrm{M}} \prod_{\mathrm{b}} \perp\right]\left(c_{(n)} \vec{A}+d_{(n)} \vec{B}\right),
\end{aligned}
$$

where $\prod_{b}$ denotes the orthogonal projection on the beam element and $\prod_{b} \perp$ the projection on the orthogonal plane to the beam element.

Such a response surface enables the introduction of uncertainty in modelling versus $C_{\mathrm{D}}$ and $C_{\mathrm{M}}$, and also marine growth effects through an increase of the beam element diameter $\left(\theta_{\mathrm{mg}}\right)$. Let $B=(\mathrm{NP})$ be a beam element. The nodal forces $\Gamma_{\mathrm{NP}}$ at node $\mathrm{N}$ (within resultant $T_{\mathrm{NP}}$ and moment $M_{\mathrm{NP}}$ ) and $\Gamma_{\mathrm{PN}}$ at node $\mathrm{P}$ equilibrate the beam element $B$ submitted to the hydrodynamic distributed forces $\left(f_{\mathrm{D}}+f_{\mathrm{I}}\right)$. By means of applying a beam finite element model, and using the kinematics energy theorem to translate the beam equilibrium, the following equation is obtained:

$\int_{\Sigma} \vec{U}(M, t) \cdot f(M) \mathrm{d} M=\left(U_{\mathrm{N}}, \Gamma_{\mathrm{NP}}\right)+\left(U_{\mathrm{P}}, \Gamma_{\mathrm{PN}}\right)$,

where $U$ denotes the displacement field and (, ) the product or co-moment of two operators.

Applying Newton's law to a homogeneous and isotropic beam element, and assuming a linear elastic behaviour under static loadings, a finite element modelling of the displacement field is deduced. Using a quadrature formulae by cutting the beam element $B$ into $n_{\mathrm{b}}$ subdivisions $\left(Q_{i} Q_{i+1}\right)$ where $Q_{1}=N$ and $Q_{n_{\mathrm{b}}}=\mathrm{P}$, then the set of Eqs. (13)-(15) leads to an explicit response surface for the nodal forces at node $\mathrm{N}, \Gamma_{\mathrm{NP}}$. The components of the nodal forces are the resultant $T_{\mathrm{NP}}(16)$ and the momentum $M_{\mathrm{NP}}$ (17). $T_{\mathrm{NP}}$ and $M_{\mathrm{NP}}$ are "the summation" in terms of finite element analysis along the beam of the distributed loading acting on this beam. The resultant has the form

$$
\begin{aligned}
T_{\mathrm{NP}}= & \frac{l}{2} \rho D \theta_{\mathrm{mg}} \sum_{i=0}^{n_{\mathrm{b}}}\left(\sqrt{a_{(n)}^{2}+b_{(n)}^{2}}\right)_{(i)} \\
& \times\left[\pi C_{\mathrm{X}} c_{11}^{(i)} \prod_{\mathrm{b}}+C_{\mathrm{D}} c_{22}^{(i)} \prod_{\mathrm{b}} \perp\right]\left(a_{(n)} \vec{A}+b_{(n)} \vec{B}\right)_{(i)} \\
& +\frac{l}{2} \pi \rho D^{2} \theta_{\mathrm{mg}}^{2} \sum_{i=0}^{n_{\mathrm{b}}}\left[C_{\mathrm{X}}^{\prime} c_{11}^{(i)} \prod_{\mathrm{b}}+C_{\mathrm{M}} c_{11}^{(i)} \prod_{\mathrm{b}} \perp\right] \\
& \times 5\left(c_{(n)} \vec{A}+d_{(n)} \vec{B}\right)_{(i)},
\end{aligned}
$$

where $c_{11}^{(i)}$ and $c_{22}^{(i)}$ are the diagonal terms of influence structural matrices deduced by integrating the classical 
shape functions of beam finite element theory, and (i) means the value of the expression at the point $Q_{i}$.

The momentum is given by

$$
\begin{aligned}
M_{\mathrm{NP}}= & \frac{l^{2}}{2} \rho D \theta_{\mathrm{mg}} \sum_{i=0}^{n_{\mathrm{b}}}\left(\sqrt{a_{(n)}^{2}+b_{(n)}^{2}}\right)_{(i)} \\
& \times\left[C_{\mathrm{D}} c^{\prime(i)} \Lambda_{\mathrm{b}}\right]\left(a_{(n)} \vec{A}+b_{(n)} \vec{B}\right)_{(i)} \\
& +\frac{l^{2}}{2} \pi \rho D^{2} \theta_{\mathrm{mg}} \sum_{i=0}^{n}\left[C_{\mathrm{M}} c^{\prime(i)} \Lambda_{\mathrm{b}}\right] \\
& \times\left(c_{(n)} \vec{A}+d_{(n)} \vec{B}\right)_{(i)},
\end{aligned}
$$

where $\Lambda_{\mathrm{b}}$ is the anti-symmetric operator with axis supported by the beam element.

It is demonstrated that the transfer function from distributed forces to concentrated nodal forces keeps the linear dependence on deterministic vectors $\vec{A}$ and $\vec{B}$. Within the value of $n_{\mathrm{b}}$ this response surface introduces a second level of complexity to be discussed (see Section 4). The set of nodal forces $\Gamma_{\mathrm{N}}$ at node $\mathrm{N}$ is then obtained as a summation of the elementary forces due to all elements connected to $\mathrm{N}$,

$\Gamma_{\mathrm{N}}=\sum_{(\mathrm{PN})} \Gamma_{\mathrm{NP}}+\Gamma_{\mathrm{O}}^{\mathrm{N}}$

This matrix response function depends on a set of seven basic variables $\left(H, T, C_{\mathrm{D}}, C_{\mathrm{M}}, C_{X}, C^{\prime}{ }_{X}, \theta_{\mathrm{mg}}\right)$ that are quite different in nature:

- the couple $(H, T)$ represents the intrinsic randomness of the wave process and includes the energetic loading of the sea;

- the hydrodynamic coefficients $C_{\mathrm{D}}, C_{\mathrm{M}}, C_{\mathrm{X}}, C^{\prime}{ }_{\mathrm{X}}$ resume the physics of the wave-structure interaction and must be seen as variables of modelling uncertainty;

- $\theta_{\mathrm{mg}}$ is a variable that represents the lack of knowledge on the increase of beam diameter caused by marine growth.

Moreover, this response function enables discussion of the complexity level to be introduced in modelling through the kinematics field model (Stokes order $n$ ), the energetic equivalence theorem (number of beam subdivisions $n_{\mathrm{b}}$ ) and the Morison equations. This last point is treated in a number of works, which suggests linearization of the drag component by using Bolotin's [35] or Caughey's [36] procedures. The polynomial expansion is then obtained by minimizing the variance of the residuals. Note that, practically, only the matrix used actually for the computation of loading on framed structures has to be changed when considering this model. The finite element modelling of the structure provides a solution expressed as a function of basic variables. Reliability analysis can be considered as a post-treatment of this solution.
The next section describes, with illustrative supports, the uncertainty and sensitivity studies needed to ensure the shape (complexity level) and the robustness of the response surface (distribution effects, goodness-of-fit measure).

\section{Uncertainty and sensitivity analysis}

The response surface presented in the previous sections is built around the transfer of the energy of the wave kinematics fields due to Morison equations. It leads to the discussion of uncertainty modelling in the model of kinematics field of water particles and the sensitivity of the transfer of its energy to complexity level. To this issue, this section points out several key points in more detail:

- uncertainty study for the stochastic kinematics fields modelling,

- complexity level in the Stokes model,

- complexity level in the structural beam element model and

- sensitivity studies for computed forces.

Finally, the robustness of the proposed surface response is shown within an illustration by comparison of distribution tails transfer obtained with the common polynomial formalism and the proposed physical function. The model presented in Section 3 is mainly based on a geometrical sight. Thus, it is well adapted for a linear algebra programming such as in Matlab (Matrix Laboratory). It is an interactive system of which the elementary data element is a matrix that does not require any dimensioning. Stochastic modelling and sensitivity analysis are consequently tractable at lower computational costs. Simulation analysis and corresponding post-processing graphics can be made in an easy-to-use environment.

The flowchart of the successive programs that is developed to perform uncertainty and sensitivity studies is presented in Fig. 4. These several steps allow control of the response.

\subsection{Description of variables and sampling techniques}

The stochastic nature of wave loading and sensitivity analysis is studied within an illustration using data modelling from the North Sea. Waves were measured by a radar distance meter on the QP structure, a four-legged jacket located between the Shetland Islands and Norway in nearly $100 \mathrm{~m}$ depth. The modelling of extreme values deduced from these measurements is detailed in $[37,38]$ : it is based on properties of Gaussian time-variant processes with assumptions of narrow-band, zero-mean, ergodicity and stationarity. It has been shown that the probability density function of extreme values can be deduced knowing the significant wave height and the duration of stationarity. Tests for validation are available in [37]. As shown in Section 3 and from the dispersion equation, the couple of random variables $(H, T)$ is obviously sufficient to compute 


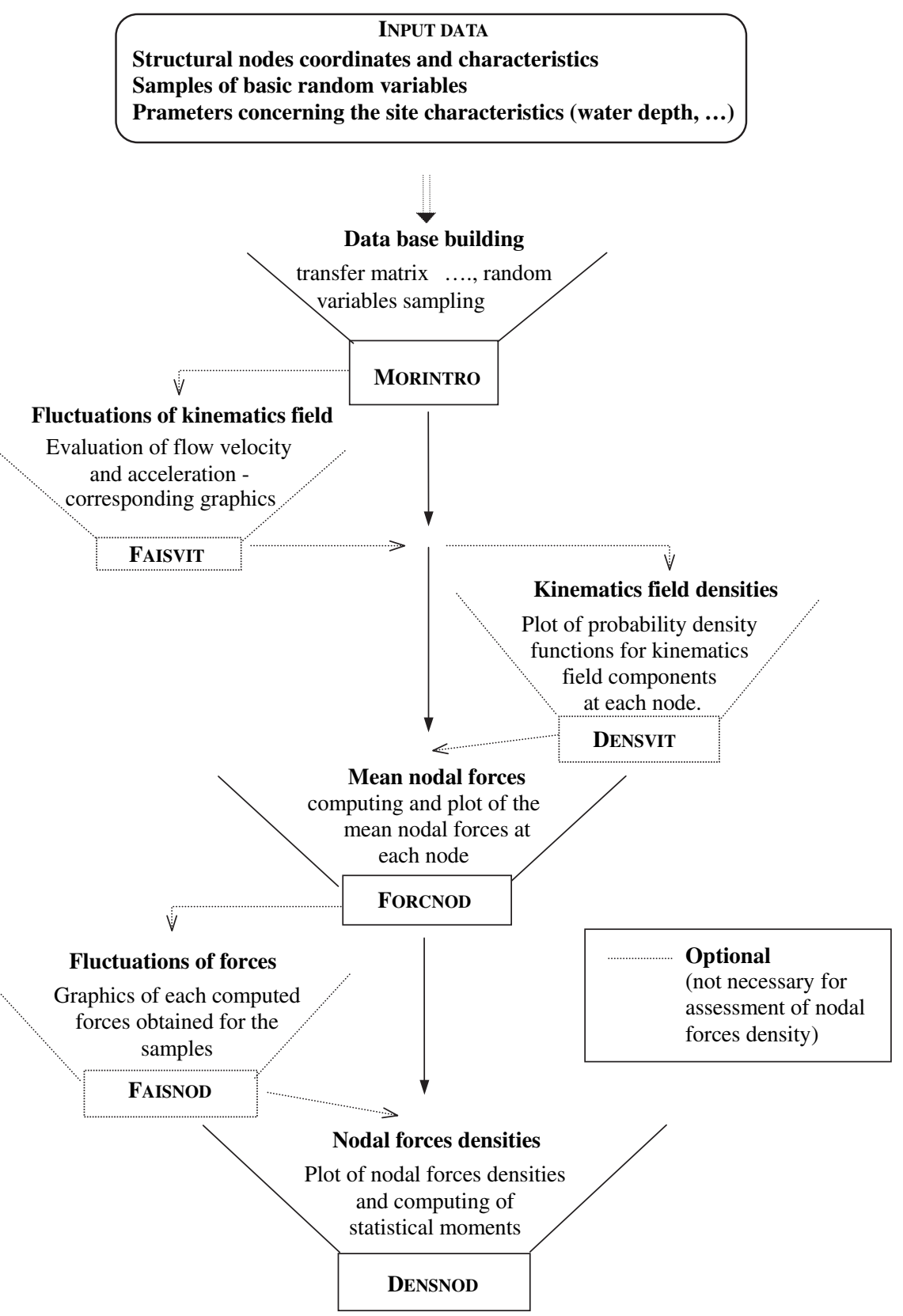

Fig. 4. Flowchart of the successive Matlab programs.

the wave velocity. Table 1 presents the probabilistic modelling of extreme waves deduced from modelling of North Sea conditions in the Frigg Field. Table 2 presents the main statistics for the basic variables.

Using these North Sea data, the Latin hypercube sampling [39] and calibration tests for the point estimate method have been performed to generate preliminary samples from the joint probability distribution for $(H, T)$ of the sea state [40]. This sampling technique provides a full coverage of the range of each input variable, but it remains questionable how far the selected samples are
Table 1

Extreme sea-state parameters

$H_{\mathrm{s}}$ : significant wave height: Gumbel distribution: $\exp (-\exp -(x-u) / \alpha)$ (ex: 100 years period: $u=15.5 \mathrm{~m} ; \alpha=1.2$ )

$T_{\text {stat }}$ : sea state duration

Exponential distribution with expectation $3 \mathrm{~h}$

$E\left(T_{\mathrm{z}} \mid H_{\mathrm{s}}\right)=\phi\left(H_{\mathrm{s}}\right)\left(\operatorname{ex}: \phi\left(H_{\mathrm{s}}\right)=\operatorname{sqrt}\left(8 H_{\mathrm{s}}+21\right)\right)$

$H$ given $\left(H_{\mathrm{s}}, T_{\text {stat }}\right):$ Gumbel distribution: $\exp (-\exp -(x-u) / \alpha)$ $u=H_{\mathrm{s}} \operatorname{sqrt}(r / 2), \alpha=H_{\mathrm{s}} / 2 \operatorname{sqrt}(2 r), r=\log \left(T_{\mathrm{stat}} / \phi\left(H_{\mathrm{s}}\right)\right)$

(ex: 100 years; $H_{\mathrm{s}}=15.5 \mathrm{~m} ; T_{\text {stat }}=3 \mathrm{~h} ; u=28.5 ; \alpha=1.9$ )

$T$ given $H$ : normal or lognormal distributed

$E(T \mid H)=\phi(H),\left(\right.$ ex: $\phi\left(H_{\mathrm{s}}\right)=\operatorname{sqrt}(6 H+39)$ 
Table 2

Hypothesis for basic variables sampling

\begin{tabular}{|c|c|c|c|c|}
\hline Basic variables & Distribution type & Conditioning & C.O.V & Truncature \\
\hline Ext. wave height, $H$ & Gumbel & Given $\left(H_{\mathrm{s}}, T_{\text {stat }}, \theta\right)$ & $8 \%$ & {$[m-2 \sigma ; m+5 \sigma]$} \\
\hline Ext. wave period, $T$ & Lognormal & Given $H$ & $10 \%$ & $\begin{array}{l}{[m-k \sigma ; m+k \sigma]} \\
k=3\end{array}$ \\
\hline $\begin{array}{l}\text { Hydr. coefficient, } \\
C_{\mathrm{D}} C_{\mathrm{M}}\end{array}$ & Normal vector & Given $H$ & Around 35\% & {$[m-p \sigma ; m+p \sigma]$} \\
\hline
\end{tabular}

representative of the whole underlying distribution. After tests on the sample, the optimum size for this multivariate problem is around 2000. The conditioning is a key point for reliability purposes as well as the truncature presented in the last column of Table 2 because the distribution functions are defined on infinite supports and some physically representative samples are required. On the basis of the analysis of statistics of outputs (variance, kurtosis, skewness), tests have been performed to define the physically suitable truncature. These results can be found in [40].

\subsection{Uncertainty study for the stochastic kinematics field modelling}

The flowchart presented in Fig. 2 enables a step-by-step system analysis for uncertainty and complexity studies to be performed. The basis of the transfer is concerned first: the stochastic nature of the kinematics field. The work focuses on velocities and deduced drag forces. The stochastic modelling is first analysed considering the first order of the Stokes model presented in (4). The intensity of the velocity vector $\vec{V}_{1}$ equals $\lambda_{1}$. It is a stochastic process indexed by $x, z$ and $t$. Abscissa $x$ is the dimensionless abscissa along the wave direction; it takes the value 0 at the beginning of the structure along the wave direction and the value 1 at the end of the structure. Index $z$ is the dimensionless profile index; it takes the value 0 at the bottom of the sea, the value 1 at the mean sea level and more in the wave area up to the mean sea level. In the considered site, values of $z$ up to 0.8 refer to the splash zone. Index $t$ is the time index; for illustrations $t=0$ (e.g. initial time) is assumed and the wave crest is on the first pile of the structure. Considering first the stochastic process $\lambda_{1}$ $(x, z, t)$ for the position at $x=0$, a process $\lambda_{1}^{(1)}$, indexed by $z$ only whose probability density is given in Fig. 5 for the set $(0.2 ; 0.4 ; 0.6 ; 0.8 ; 1.0 ; 1.1)$ of values for $z$, is defined. From (7), an expression of the wave intensity module as a product of the previous process (the profile $\lambda_{1}^{(1)}$ ) and a deterministic function obtained for the expectation of the mean wave period $T$ (see Eq. (19)) is deduced. This function is $D_{1}$.

$\lambda_{(1)}(x, z, t)=\underline{D_{1}}(x, z, t) \lambda_{(1)}^{(1)}(z)$,

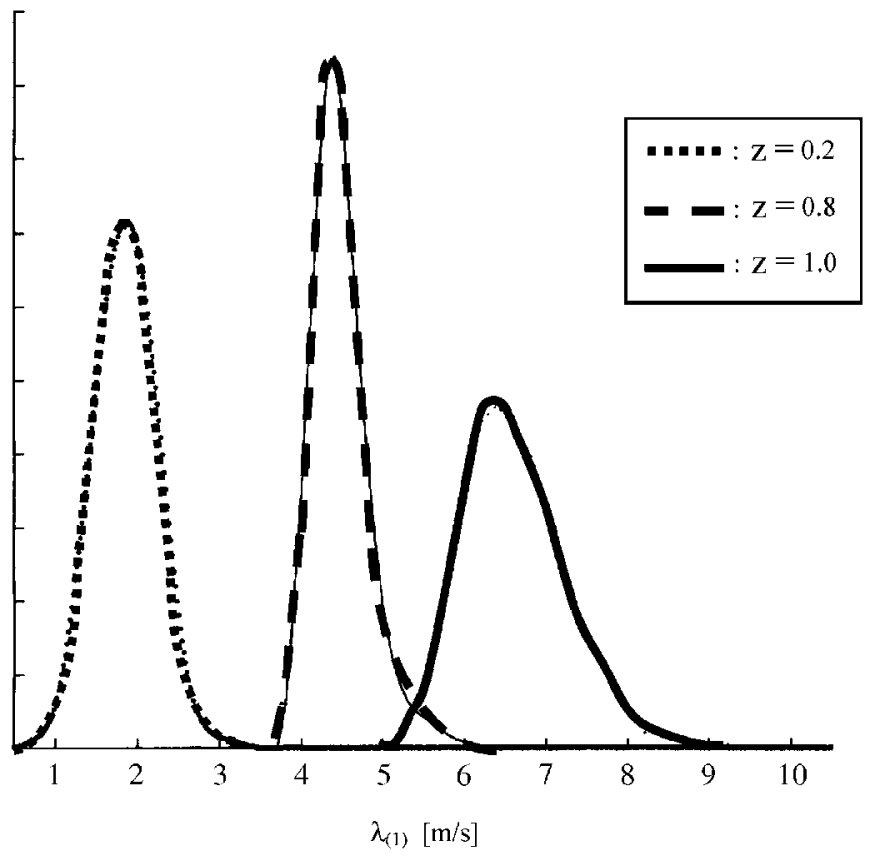

Fig. 5. Probability density function of $\lambda_{(1)}(O, z, 0)$.

where

$D_{1}=\sqrt{1-\frac{\sin ^{2}\left(k l x-\psi_{t}\right)}{\cosh ^{2}(k d z)}}$.

The variation of the deterministic scalar function $D_{1}$ $(x, z, 0)$ is plotted in Fig. 6 upon $x$ for three values of $z$. It explains some expansions around the mean wave number. So using this linear relationship, it is quite easy to achieve the stochastic variation of the velocity intensity module $\lambda_{(1)}$. It is interesting to validate this result through a comparative study of the stochastic fluctuations for the Airy wave intensity module using simulation. Fig. 7 enables comparison of the density of the wave intensity module obtained from the previous linear relationship (Eq. (14)) and from a complete simulation. They are plotted in the plane $(x, z)$ for the same set of $z$ values $(0.2 ; 0.8 ; 1.0)$ selected for Fig. 6. There is no discrepancy in the splash area $(z>0.8)$ where the forces are significant. The distribution is further tightened near the bottom, but 
the approximation remains quite good because of the low level of forces at this depth. Suppose now $t \neq 0$; the assertions remain true once $\underline{D_{1}}(x, z, 0)$ is extended to $\underline{D_{1}}(x, z, t)$.

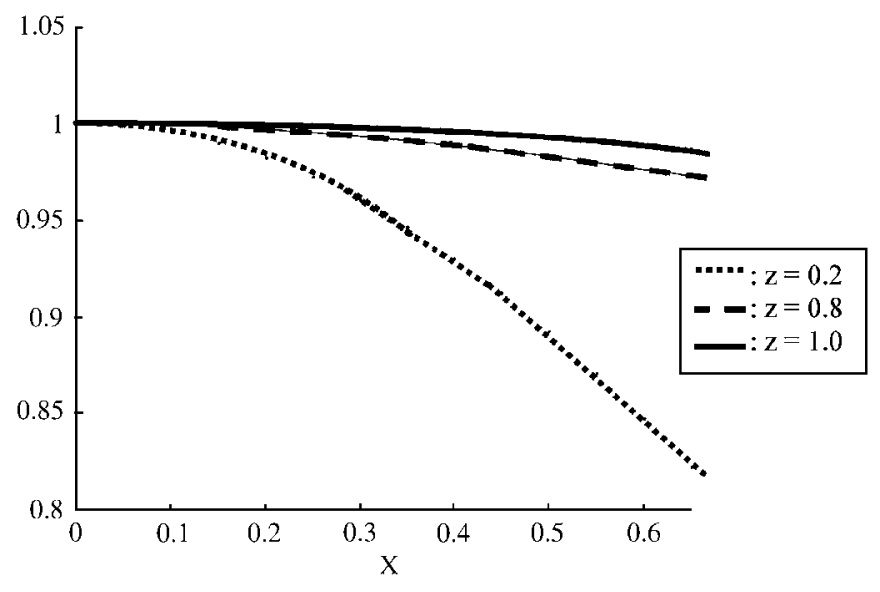

Fig. 6. Deterministic scalar function $D_{(1)}(x, z, 0)$.

\subsection{Complexity level in the Stokes model}

Eq. (12) generalizes the formal modelling for the $n$th Stokes model. To keep the physical meaning of the wave kinematics modelling and to reduce the computational costs (see Section 3.1), there is a need to discuss the complexity level to introduce in the stochastic model. First consider the role of the second order into the Stokes model. Following the guidelines of the previous section, the stochastic velocity intensity is governed by profile functions. Thus, Fig. 8 presents variations of statistics for the intensity of the first and second order upon $z$, respectively $\lambda_{(1)}$ and $\lambda_{(2)}$. The profile at the mean value, at the median and at plus/minus one and two times the standard deviation around the mean are plotted. Statistics of $\lambda_{(2)}$ are decreasing so quickly with depth that the discrepancy between the second and the first order is quite negligible. Around the mean sea level, the statistics of $\lambda_{(2)}$ are larger but they remain at a small percentage of the $\lambda_{(1)}$ ones. This example is obviously site specific but it shows that the

\section{Linear relationship}

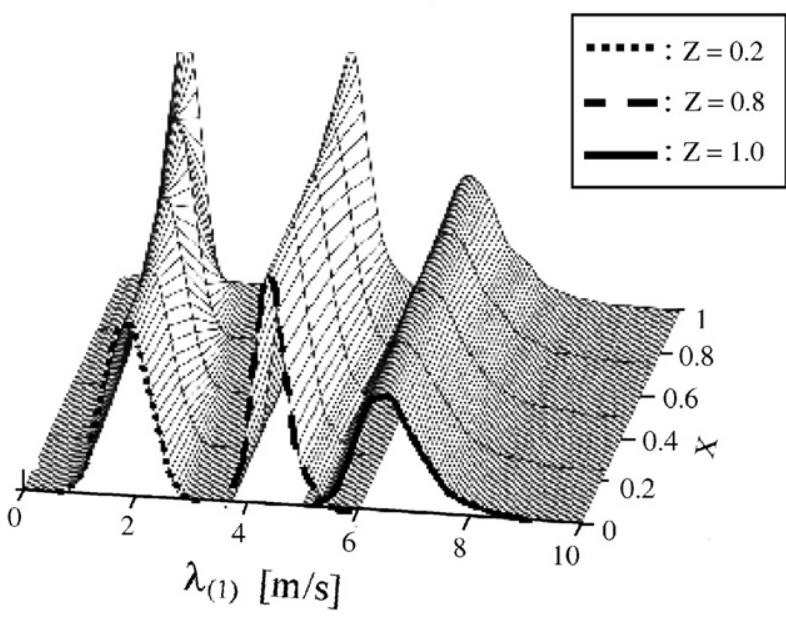

Complete simulation

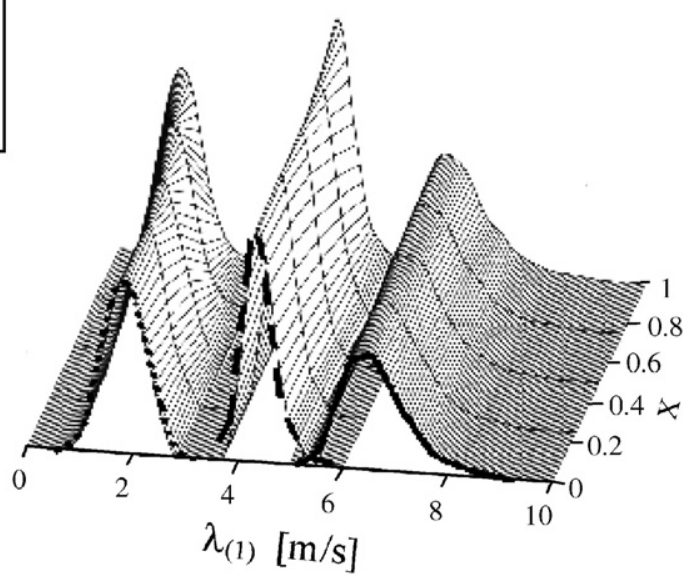

Fig. 7. Discrepancy analysis of densities for the stochastic process $\lambda_{(1)}(x, z, t)$.
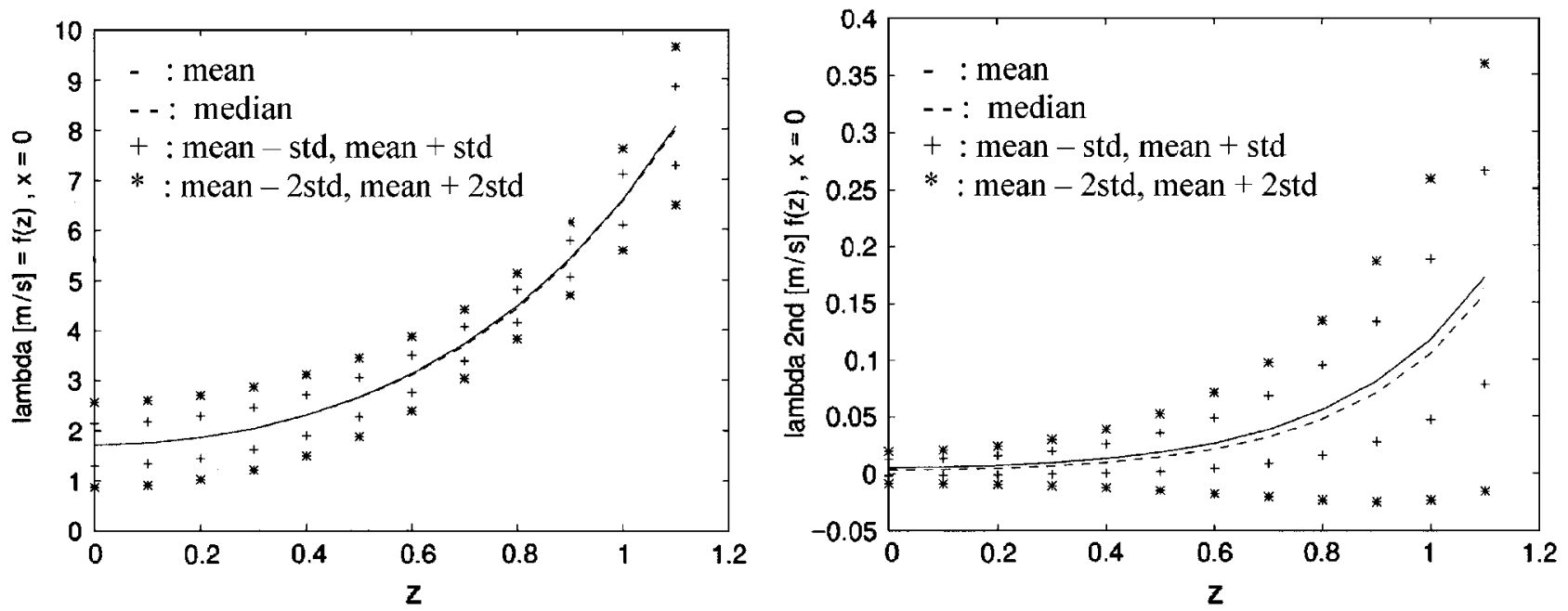

Fig. 8. Statistics of $\lambda_{(1)}(x, z, t)$ and $\lambda_{(2)}(x, z, t)$ as profile functions. 
increase of the complexity level in stochastic modelling has to be viewed with care. It is a time-consuming option when computing, and which does not give necessarily more realistic results.

\subsection{Complexity level in the Morison equation approximation}

Section 3.2.3 presents the opportunity to linearize the drag component of the Morison equation. This procedure is usual [26]. Table 3 gives the results for both approximation models: Caughey's procedure and the proposed response surface. In this example, the main basic variables are the wave height $H$ and the wave period $T$. The normalized variance of the residuals is practically the same whatever the model. The discrepancy between the partial derivatives is larger. This explains why the statistics of high order are better transferred through the physical response surface than under a polynomial expansion. It is also noticed that the proposed metrics provide a more robust measure of the goodness of fit for constructing response surfaces.
Following the flowchart described in Fig. 2, distributions of concentrated nodal force intensity are computed for the North Sea site. The intensity of the resultant $T_{\mathrm{BA}}$ at node B of hydrodynamic loading acting on the beam $\mathrm{A}-\mathrm{B}$ is considered (see Fig. 12). Under the linearization assumption, the distribution is tightened and becomes symmetrical (see Fig. 9). In this case, it leads to conservative results by comparison with the physical model. This confirms the distribution effects as a key point in response surface modelling.

\subsection{Complexity level in structural modelling}

Eqs. (16) and (17) give the quadrature formula used to compute the equivalent nodal forces. Then, the question of the optimization of the number $n_{\mathrm{b}}$ of beam subdivisions must be answered. Consider a vertical beam at the position $x=0$ with diameter $1.2 \mathrm{~m}$, length $20 \mathrm{~m}$ and the median section at the mean sea level. The profile of drag forces, i.e. those calculated from the flow velocity, is presented in Fig. 10 for two numbers of subdivisions, 2 and 10 . The corresponding densities of probability for the equivalent

Table 3

Normalized error variances ( $\tilde{f}$ denotes the approximation, $f$ the true response)

\begin{tabular}{lllr}
\hline Approximation format & $\operatorname{Var}[\tilde{f}-f] / \operatorname{Var}[f]$ & $\operatorname{Var}[\partial(\tilde{f}-f) / \partial H] / \operatorname{Var}[\partial f / \partial H]$ & $\operatorname{Var}[\partial(\tilde{f}-f) / \partial T] / \operatorname{Var}[\partial f / \partial T]$ \\
\hline Response surface (Eq. (4)) & $13 \%$ & $24 \%$ & $3 \%$ \\
Polyn. expansion [12] & $11 \%$ & $55 \%$ & $26 \%$ \\
\hline
\end{tabular}
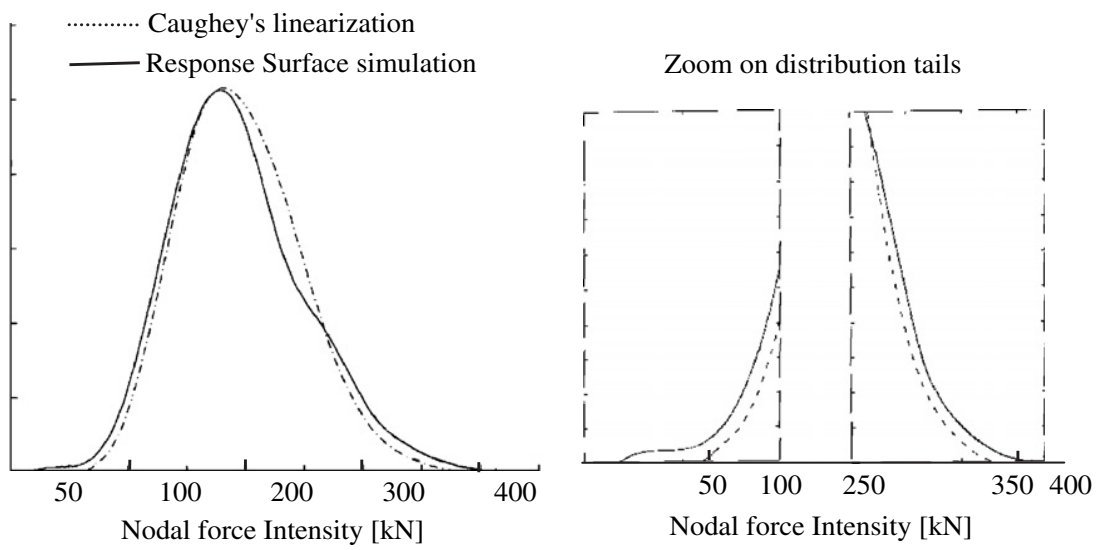

Fig. 9. Distribution effects on the intensity of the resultant $T_{\mathrm{BA}}$ at node B due to linearization.

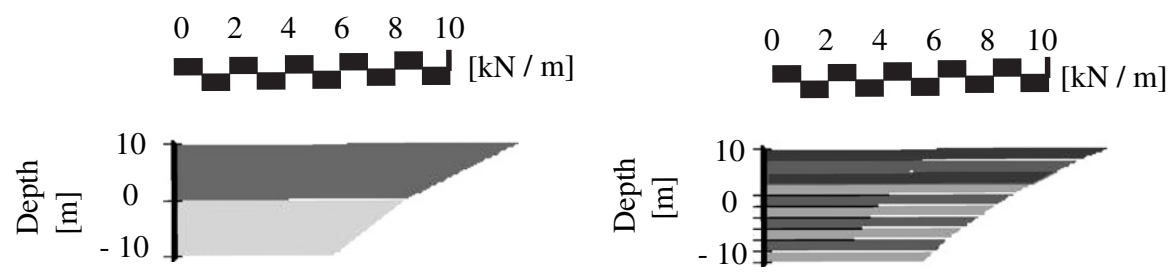

Fig. 10. Drag forces on vertical component for 2 and 10 subdivisions. 
nodal forces at the node placed at depth $10 \mathrm{~m}$ up to the mean sea level are plotted in Fig. 11. Distributions are very similar especially in the zone of reliability interest, i.e. the distribution tails. For this site, a subdivision of 2 is therefore recommended.

\subsection{Sensitivity studies for computed forces}

Sensitivity studies are recommended for analysing problems in which large uncertainties occur and where

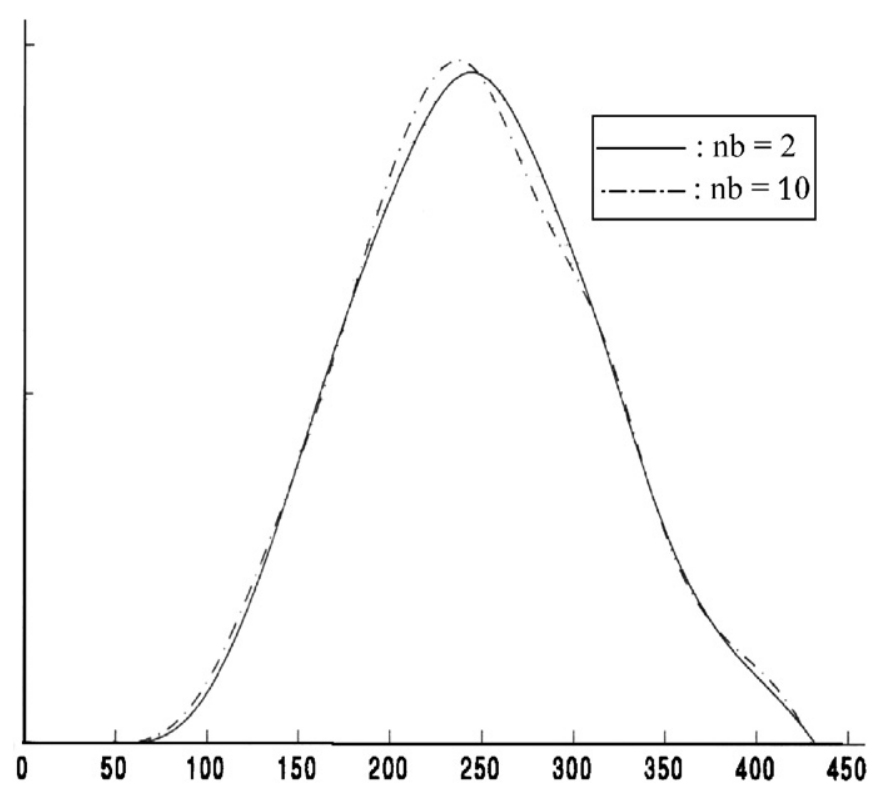

Fig. 11. Densities of nodal forces at depth $10 \mathrm{~m}$ for 2 and 10 subdivisions.

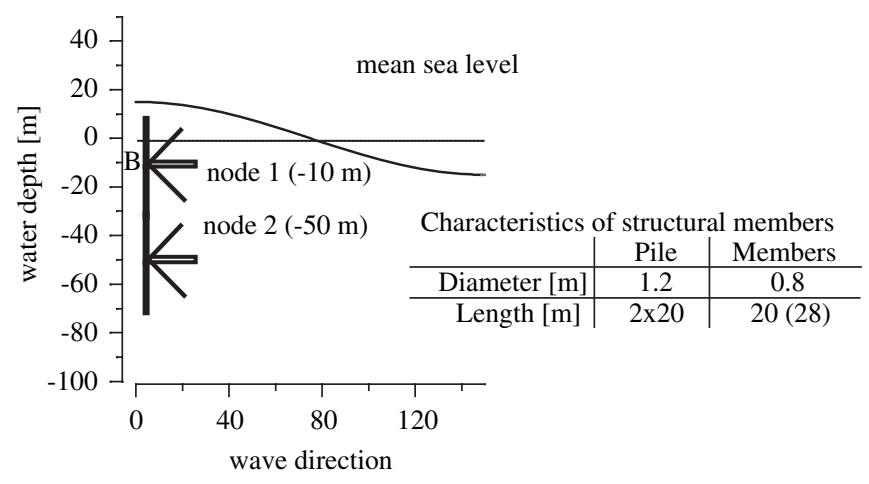

Fig. 12. Location and characteristics of nodes 1 and 2. the transfer functions are non-linear. It allows ranking of the variables and to select those with a major contribution to the response fluctuations. This question is discussed with two techniques:

i. The Monte-Carlo analysis starts from a pseudo-sampling approach to represent the system inputs.

ii. The differential analysis is very useful when explicit formulations are provided such as those proposed by the RSM.

The output considered here is the resultant of equivalent nodal forces at two nodes, each placed at the connection of five beams (see Fig. 12). Inputs (environmental conditions) have been described previously in Tables 1 and 2 and the structural characteristics are presented in Fig. 12. Statistics (mean and coefficient of variations) obtained with the first technique (i) are presented in Table 4 . This technique leads of course to a bias in mean estimation. The main contributors are obtained among the basic variables to the response by the corresponding contribution to the output statistical dispersion. The main variables are clearly the wave height $H$ and the drag coefficient $C_{\mathrm{D}}$. It is noticed that the wave period acts upon when the water depth index increases.

The second technique (ii) can be used for ranking the basic variables and for evaluating their respective contribution to the total response variance $V(f)$ of the response function $f$ under investigation. The second-order Taylor expansion is in the form

$$
\begin{aligned}
f(X)= & f\left(X_{0}\right)+\sum_{j=1}^{6}\left[\frac{\partial f\left(X_{0}\right)}{\partial X_{j}}\right]\left(X_{j}-X_{j 0}\right) \\
& +\frac{1}{2} \sum_{j=1}^{6} \sum_{k=1}^{6}\left[\frac{\partial^{2} f\left(X_{0}\right)}{\partial X_{j} \partial X_{k}}\right]\left(X_{j}-X_{j 0}\right)\left(X_{k}-X_{k 0}\right)
\end{aligned}
$$

and the variance is deduced:

$$
\begin{aligned}
V(f)= & \sum_{j=1}^{n}\left[\frac{\partial f\left(X_{0}\right)}{\partial X_{j}}\right]^{2} V\left(X_{j}\right) \\
& +\sum_{j=1}^{6}\left[\frac{\partial f\left(X_{0}\right)}{\partial X_{j}}\right]\left[\frac{\partial^{2} f\left(X_{0}\right)}{\partial X_{j}^{2}}\right] \mu_{3}\left(X_{j}\right) \\
& +[\text { covariance terms }],
\end{aligned}
$$

where $n$ is the number of basic random variables, $\mu_{3}$ is the skewness and $X_{0}$ is the vector of the mean values of

Table 4

Sensitivity analysis on nodal forces ( $m$, mean value; $v$, coefficient of variation)

\begin{tabular}{llcccc}
\hline & All random & $\begin{array}{l}\text { All determinist. } \\
\text { except } H \text { and } C_{\mathrm{D}}\end{array}$ & \multicolumn{2}{c}{$\begin{array}{l}\text { All random except } H \\
\text { and } C_{\mathrm{D}}\end{array}$} & $\begin{array}{l}\text { All determinist. } \\
\text { except } T\end{array}$ \\
\hline Node 1 & & 458.5 & 459.5 & 459 & 458 \\
& $\mathrm{~m}(\mathrm{kN})$ & $26.5 \%$ & $26.5 \%$ & $6 \%$ & $6 \%$ \\
Node 2 & $v$ & 118 & 115.5 & 116.5 & 116.5 \\
& $\mathrm{~m}(\mathrm{kN})$ & $33.5 \%$ & $26.5 \%$ & $13.5 \%$ & $13.5 \%$ \\
\hline
\end{tabular}


Table 5

Influence ratios into the total response variance

\begin{tabular}{lllrl}
\hline Node no. & $H$ & $C_{\mathrm{D}}$ & $T$ & Total \\
\hline 1 & $35 \%$ & $61 \%$ & $3 \%$ & $99 \%$ \\
2 & $27 \%$ & $40 \%$ & $32 \%$ & $99 \%$ \\
\hline
\end{tabular}

variables $X_{j}$. Considering the first order of Taylor expansion, the influence ratio is simply defined by formula (22), if the covariance terms can be neglected:

$\tau_{i}=\left[\frac{\partial f\left(X_{0}\right)}{\partial X_{j}}\right]^{2} \frac{V\left(X_{j}\right)}{V(f)}$.

Table 5 presents influence ratios (e.g. the contribution of each variable to the total output variance). This confirms the results previously obtained. It gives more precisely a quantitative measure of the relative importance for each variable.

The results obtained through both techniques (MonteCarlo simulations and differential analysis) are complementary and give a robust aid tool for variable ranking and strategy for uncertainty analysis. It appears that the modelling of variables $H$ and $C_{\mathrm{D}}$ (probability density function, correlation, conditioning, etc.) is, for this site, of utmost importance.

\section{Conclusion}

The response surface methodology (RSM) has been developed considering mainly physical models with specific emphasis on environmental loading application. This conceptual toll has to be managed, keeping in mind its implicit consequences. In fact, results of structural reliability are very dependent on the basic modelling assumptions. Taking into account a set of guidelines for the building criteria, it enables a rational use of this methodology.

To illustrate these criteria, a matrix response surface of equivalent nodal forces due to wave loading on framed tubular structures is presented. It is built around the transfer of energy of wave kinematics. Its original geometrical format is shown to be versatile for verifying the dominant building criteria of response surfaces. Moreover, the stochastic structure of the kinematics field is specified following space/time indices to be considered. Further studies are actually developed on this basis:

- response surface of wave-current interaction,

- loading on cracked structures,

- effect of the marine-growth profile and evolution for time-variant reliability and

- wind loading on framed structures.

Finally, RSM is an efficient procedure to perform subsequent uncertainty and sensitivity studies once models with physical parameters are available. It leads one to emphasize the role of complexity level and polynomial expansion to reduce the cost of computation time. It is suitable when performing computation in a wider context of structural reliability once new methods of computing (stochastic chaos) are introduced too.

\section{References}

[1] Labeyrie J, Schoefs F. A discussion on response surface approximations for use in structural reliability. Sixth IFIP WG7.5, Reliability and optimisation of structural systems, no. 15. London: Chapman \& Hall; 1995. p. 161-8.

[2] Standing R. Wave loading on offshore structures: a review. Ocean Sci Eng 1984;9:1.

[3] Lightill J. Fundamentals concerning wave loadings on offshore structures. J Fluid Mech 1986;173.

[4] Standsby PK, Isaacson M. Recent developments in offshore hydrodynamics - workshop report. Appl Ocean Res 1987;9:3.

[5] Wilson JF. Wave forces on structures. New York: Wiley; 1984.

[6] Tickell RS, Bishop JR. Analysis of waves and wave forces at the Christchurch Bay Tower. In: Proceedings of the Offshore Mechanics and Arctic Engineering, Dallas, USA, 1985.

[7] Spidsoe N, Brahamy HP, Skjadstad O. Non linear random wave loading on fixed offshore platforms. In: Offshore technology conference, paper no. 5101, 1986.

[8] Allender JH, Petrauskas C. Measured and predicted wave plus current loading on laboratory-scale space-frame structure. In: Offshore technology conference, OTC 1987. p. 5371.

[9] Bearman PW, Chaplin JR, Graham JMR, Kostense JR, Hall PF, Klopman $\mathrm{G}$. The loading of a cylinder in post-critical flow beneath periodic and random waves. In: Proceedings of behavior of offshore structures conference, 1985. p. 213-25.

[10] Blumberg R, Rigg AM. Hydrodynamic drag at supercritical Reynolds numbers. In: ASME conference, June 1961.

[11] Eastwood JW, Watson CJH. Implications of wave-current interactions for offshore design, E\&P forum workshop, Wave and current kinematics and loading, Paris, October 1989.

[12] Garrison CJ. Drag and inertia forces on circular cylinders in harmonic flow. ASCE J Waterway, Port, Coastal Ocean Eng 1990;116(2).

[13] Heideman JC, Olsen O, Johanson P. Local wave force coefficients. In Proceedings of ASCE civil engineering in the oceans IV conference, September 1979 .

[14] Keulegan GH, Carpenter LH. Forces on cylinders and plates in an oscillating fluid. J Res Natl Bur Stand 1958;60(5).

[15] Lambranos KF, Steele KM, Finn LD. Wake and shielding effects on hydrodynamic loading. In: Proceedings of E\&P forum workshop on wave and current kinematics and loading, Paris, October 25-26, 1989.

[16] Madsen OS. Hydrodynmic forces on circular cylinders. Appl Ocean Res 1986;8(3).

[17] Manners W, Rainey RCT. Hydrodynamic forces on fixed submerged cylinders. Proc R Soc London 1992:436.

[18] Rodenbusch G, Gutierrez CA. Forces on cylinders in two-dimensional flows, vol. 1. Shell Development Company, Report No. BRC13-83, 1983.

[19] Sarpkaya T, Isaacson M. Mechanics of wave forces on offshore structures. New York: Van Nostrand Reinhold; 1981.

[20] Sterndorff MJ, Velk P, Klinting P. Coupled experimental and analytical investigation of hydrodynamic forces on a jacket in waves. In: Society for Underwater Technology, environmental forces on offshore structures and their prediction conference, 1990.

[21] Taylor PH. Current blockage: reduced forces on offshore space-frame structures. In: Offshore Technology Conference, OTC 6519, 1991. 
[22] Wolfram J, Theophanatos A. The effects of marine fouling on the fluid loading of cylinders: some experimental results. In: Offshore technology conference proceedings, OTC 4954, 1985.

[23] RASOS Theoretical Manual. Reliability methods for the design and operation of offshore structures. Program Brite P-1270, doc A. 38, 1991.

[24] Leira BJ, Holmås T, Ferfjord K. Response surface parametrization for estimation of fatigue damage and extreme response of marine structures. In: Der Kiureghian A, Madanat S, Pestana JM, editors. Ninth International conference on application of statistics and probability in civil engineering, (ICASP9), vol. 1, Theme E "Damage and deterioration". Rotterdam: Millpress; 2003. p. 589-98.

[25] Labeyrie J. Response surface methodology in structural reliability. Probabilistic methods for structural design. In: Guedes Soares C, editor. Solid mechanics and its applications, vol. 56. Dordrecht: Kluwer Academic Publishers; 1997. p. 39-58.

[26] Bouyssy V, Rackwitz R. Approximation of non-normal responses for drag dominated offshore structures. Reliability and optimization of structural systems. In: Proceedings of the sixth, IFIP WG 7.5. London: Chapman \& Hall; 1994. p. 161-8.

[27] Faravelli L. Response surface approach for reliability analysis. J Eng Mech (ASCE) 1989;115(12):2763-81.

[28] Faravelli L. Structural reliability via response surface. In: Bellomo N, Casciati F, editors. Proceedings of Iutam symposium on nonlinear stochastic mechanics. Berlin: Springer; 1992. p. 213-23.

[29] Colombi P, Faravelli L. Stochastic finite element via response surface: fatigue crack growth problems. Probabilistic methods for structural design. In: Guedes Soares C, editor. Solid mechanics ans its applications, vol. 56. Dordrect: Kluwer Academic Publishers; 1997. p. 313-38.

[30] Muzeau JP, Lemaire M, Besse P, Locci JM. Evaluation of reliability in case of complex mechanical behaviour. In: 12th International conference on Offshore Mechanics and Arctic Engineering (O.M.A.E.'93), vol. II, "Safety and reliability". ASME, 1993. p. 47-56.

[31] Labeyrie J, Schoefs F. In: Matrix response surfaces for describing environmental loads, vol. II, Safety and reliability. Proceedings of the 15th O.M.A.E., Florence, ASME, 1996. p. 119-26.

[32] Lions JL. Problèmes aux limites non homogènes et applications. Paris: Dunod Editeur; 1968.

[33] Morison JR, O'Brien MP, Johnson JW, Schaff SA. The forces exerted by surfaces waves on piles. Petroleum Trans 1950;189:149-54.

[34] Labeyrie J, Schoefs F. A formal geometrical modelling of wave actions for structural reliability, safety and reliability symp. In: Proceedings of the 14th international conference on Offshore Mechanics and Arctic Engineering (O.M.A.E.'95), Copenhagen, ASME, 1995. p. 85-90.

[35] Bolotin VV. Statistical methods in structural mechanics. San Francisco: Holden Day; 1969.

[36] Caughey TK. Equivalent linearization techniques. J Acoust Soc Am 1963;35:1706-11.

[37] Doucet Y, Labeyrie J, Thebault J. Validation of stochastic environmental design criteria in the Frigg field, in adv. Underwater Technol, SUT 1987;12:45-59.

[38] Labeyrie J. Time scales and statistical uncertainties in the prediction of extreme environmental conditions, reliability engineering and system safety. England: Elsevier Science Publishers Ltd.; 1991. p. $243-66$.

[39] Iman RL, Helton JC, Campbell JE. An approach to sensitivity analysis of computer models - Part I: introduction, input variable selection and preliminary variable assessment. J Qual Technol 1981;13(3).

[40] Schoefs F. Response surface modelling of wave loads in structural reliability analysis. PhD dissertation, Nantes, 1996, 173p. 